\title{
Esophagogastric Junction Outflow Obstruction Caused by Gabapentin
}

TO THE EDITOR: We report the case of a 65 year old woman referred for dysphagia. Her medical history included hypothyroidism treated by L-thyroxin, hypercholesterolemia treated by atorvastatin, essential tremor treated by propranolol, and a post-traumatic trigeminal neuralgia treated by $1200 \mathrm{mg}$ gabapentin per day. This treatment had been administered for 5 months with good efficacy on pain relief.

Progressively, the patient developed thoracic pain, dysphagia, and regurgitations leading to reduced food intake. Clinical examination evidenced a loss of $10 \mathrm{~kg}$. Treatment with proton pump inhibitors and trimebutine did not improve the symptoms. Laboratory testing, thoracoabdominopelvic CT scanner, esophagogastroduodenoscopy, and colonoscopy with biopsies (including esophageal biopsies) were unremarkable. A high-resolution esophageal manometry revealed a median 4-second integrated resting pressure of 20.2 $\mathrm{mmHg}$, with a minimal resting pressure of the lower esophageal sphincter of $50.6 \mathrm{mmHg}$, and $100 \%$ normotonic and peristaltic esophageal contractions induced by the wet swallows, a mean distal latency of 6 seconds, and a mean distal contractile integral of 2262 $\mathrm{mmHg} \cdot \mathrm{cm} \cdot \mathrm{sec}$ (Figure A). Barium esophagogram showed a moderately distended esophageal body and a delayed esophageal emp- tying (Figure B). Taking into account the normal (and repeated) endoscopic and scannographic investigations, we considered the possibility of a role of the gabapentin in the esophagogastric junction outflow obstruction (EGJOO) and stopped this treatment. Three months later, the dysphagia had improved, and follow-up manometry and barium swallow were normal (Figures $\mathrm{C}$ and $\mathrm{D}$ ).

Gabapentin has a recognized efficacy in the treatment of neuropathic pain, ${ }^{1}$ and has been proposed as a therapy for pharyngeal discomfort and pain during chemoradiotherapy ${ }^{2}$ or functional cervical pain. ${ }^{3}$ Gabapentin is a gamma-amino butyric acid analogue of which the exact mechanism of action remains to be determined. However, it could act as a gamma-aminobutyric acid B (GA$\mathrm{BAB})$ receptor agonist, like baclofen. ${ }^{1}$ Baclofen, along with other GABAB receptor agonists, has been proposed as a second line treatment of gastro-esophageal reflux disease, since it reduces the rate of transient lower esophageal sphincter relaxations, the rate of gastroesophageal reflux episodes and increases the basal lower esophageal sphincter pressure. ${ }^{4,5}$ In our patient case, the symptoms and high-resolution manometry measurements before and after discontinuation of gabapentin are in favor of an increased tonicity of the lower esophageal sphincter, characterized as an esophagogastric
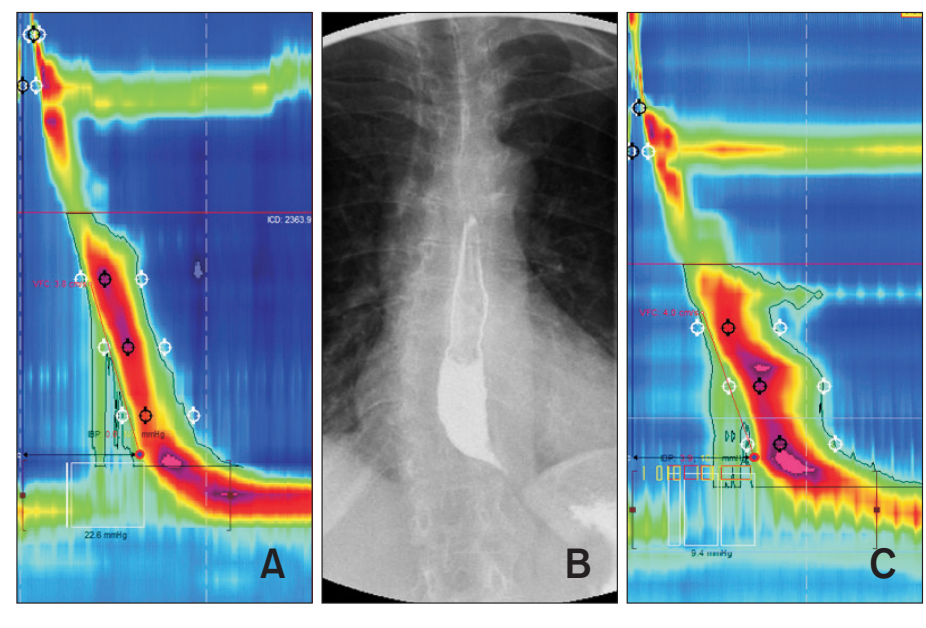

Figure. High-resolution esophageal manometry and barium esophagogram showing an esophagogastric junction outflow obstruction characterized by a normotonic persistaltic esophageal contraction associated with an elevated median 4-seconds integrated resting pressure (> $15 \mathrm{mmHg}$ ) (panel A), and a tight esophagogastric junction with mildly dilated esophagus on esophagogram (panel B). Three months later, after discontinuation of gabapentin, high-resolution manometry (panel C) as well as barium esophagogram (panel D) went back to normal. 
junction outflow obstruction according to the Chicago classification of esophageal motility disorders version $3.0^{6}$ induced by gabapentin. The recurrence of dysphagia and EGJOO after reintroduction of gabapentin would have further supported this hypothesis but was however not performed in this patient. EGJOO is a manometric entity including a variety of conditions. Usually, the patient workup includes, as in our patient case, repeat endoscopy and CT scanner to rule out esophageal stenosis, hiatal hernia, and tumoral infiltration of the esophagogastric junction; and going through the patient medications, to check for the absence of any opioid-derived treatment capable of inducing such manometric features. Our patient case suggests that gamma-amino butyric acid analogues such as gabapentin should belong to the list.

\section{Maximilien Barret, ${ }^{1,2 *}$ Marie-Anne Guillaumot, ${ }^{1,2}$ Chloé Léandri, ${ }^{1,2}$ Raphael Gaillard, ${ }^{3,4,5,6}$ and Stanislas Chaussade ${ }^{1,2}$ \\ ${ }^{1}$ Hôpital Cochin, Service de gastro-entérologie, Assistance Publique- Hôpitaux de Paris, Paris, France; ${ }^{2}$ Université Paris Descartes, Sorbonne Paris Cité, Paris, France; ${ }^{3}$ Centre Hospitalier Sainte-Anne, Service Hospitalo-Universitaire, Paris, France; ${ }^{4}$ Centre de Psychiatrie et Neurosciences, INSERM UMR 894, CNRS, Paris, France; ${ }^{5}$ Institut de Psychiatrie (GDR 3557), Paris, France; and ${ }^{6}$ Université Paris Descartes, Sorbonne Paris Cité, Paris, France}

1. Sills GJ. The mechanisms of action of gabapentin and pregabalin. Curr Opin Pharmacol 2006;6:108-113.

2. Starmer HM, Yang W, Gourin CG, et al. One-year swallowing outcomes in patients treated with prophylactic gabapentin during radiationbased treatment for oropharyngeal cancer. Dysphagia 2017;32:437-442.

3. Kirch S, Gegg R, Johns MM, Rubin AD. Globus pharyngeus: effectiveness of treatment with proton pump inhibitors and gabapentin. Ann Otol Rhinol Laryngol 2013;122:492-495.

4. Grossi L, Spezzaferro M, Sacco LF, Marzio L. Effect of baclofen on oesophageal motility and transient lower oesophageal sphincter relaxations in GORD patients: a 48-h manometric study. Neurogastroenterol Motil 2008;20:760-766.

5. Boeckxstaens GE, Beaumont H, Hatlebakk JG, et al. A novel reflux inhibitor lesogaberan (AZD3355) as add-on treatment in patients with GORD with persistent reflux symptoms despite proton pump inhibitor therapy: a randomised placebo-controlled trial. Gut 2011;60:1182-1188.

6. Kahrilas PJ, Bredenoord AJ, Fox M, et al. The Chicago Classification of esophageal motility disorders, v3.0. Neurogastroenterol Motil 2015;27:160-174.

\section{Financial support: None.} Conflicts of interest: None.

Author contributions: Maximilien Barret drafted the manuscript, Marie-Anne Guillaumot, Chloé Léandri, Raphael Gaillard, and Stanislas Chaussade analyzed the data and provided critical review to the manuscript. All authors approved the final version of the paper. 